\title{
Road Network Reconstruction from satellite images with Machine Learning Supported by Topological Methods
}

\author{
Tamal K. Dey, Jiayuan Wang, Yusu Wang* \\ Department of Computer Science and Engineering, The Ohio State University \\ Columbus, Ohio
}

\begin{abstract}
Automatic Extraction of road network from satellite images is a goal that can benefit and even enable new technologies. Methods that combine machine learning (ML) and computer vision have been proposed in recent years which make the task semi-automatic by requiring the user to provide curated training samples. The process can be fully automatized if training samples can be produced algorithmically. In this work, we develop such a technique by infusing a persistence-guided discrete Morse based graph reconstruction algorithm into ML framework. We elucidate our contributions in two phases. First, in a semi-automatic framework, we combine a discrete-Morse based graph reconstruction algorithm with an existing $\mathrm{CNN}$ framework to segment input satellite images. We show that this leads to reconstructions with better connectivity and less noise. Next, in a fully automatic framework, we leverage the power of the discrete-Morse based graph reconstruction algorithm to train a CNN from a collection of images without labelled data and use the same algorithm to produce the final output from the segmented images created by the trained CNN. We apply the discrete-Morse based graph reconstruction algorithm iteratively to improve the accuracy of the CNN. We show experimental results on datasets from SpaceNet Challenge. Full version of the paper appears in [8]
\end{abstract}

\section{CCS CONCEPTS}

- Information systems $\rightarrow$ Geographic information systems; - Computing methodologies $\rightarrow$ Image segmentation.

\section{KEYWORDS}

map generation, satellite images, machine learning, topological method, Morse complex

\section{ACM Reference Format:}

Tamal K. Dey, Jiayuan Wang, Yusu Wang. 2019. Road Network Reconstruction from satellite images with Machine Learning Supported by Topological Methods. In 27th ACM SIGSPATIAL International Conference on Advances in Geographic Information Systems (SIGSPATIAL '19), November 5-8, 2019, Chicago, IL, USA. ACM, New York, NY, USA, 4 pages. https: //doi.org/10.1145/3347146.3359348

*tamaldey,yusu@cse.ohio-state.edu,wang.6195@osu.edu. This work is partially sup ported by NSF under grants CCF-1740761, RI-1815697, CCF-1733798 and CCF-1618247.

Permission to make digital or hard copies of part or all of this work for personal or classroom use is granted without fee provided that copies are not made or distributed for profit or commercial advantage and that copies bear this notice and the full citation on the first page. Copyrights for third-party components of this work must be honored

For all other uses, contact the owner/author(s).

SIGSPATIAL '19, November 5-8, 2019, Chicago, IL, USA

(C) 2019 Copyright held by the owner/author(s)

ACM ISBN 978-1-4503-6909-1/19/11.

https://doi.org/10.1145/3347146.3359348

\section{INTRODUCTION}

Layout of road networks is essential for diverse applications in geographic information systems. Efficient reconstruction from images and timely updates of road networks are important both for map designs and handling events such as natural disasters. The availability of high-resolution satellite images has enabled such technology in recent years though the process is not fully automatic. Currently the road extraction from satellite images is mainly completed manually [19]. Doing so automatically or even semi-automatically in a reliable manner is challenging as there are a variety of different types of roads whose images are cluttered with noise and occlusions (by cars/trees etc).

Extracting lane-related information from high resolution satellite images has been addressed in recent years [11, 18, 22]. Specifically for road extraction, a range of methods that combine machine learning and computer vision methods have been proposed to reconstruct roads using labelled data. These are semi-automatic in the sense that they use manually curated samples to train the classifier. These methods often consist of two main stages: background segmentation and centerline extraction. The background segmentation is usually done via machine learning methods such as performing feature extraction and pixel-wise label predictions with SVM $[4,16]$ or CNN [3]. The baseline algorithms for SpaceNet Challenge [19] use the architecture such as U-Net[15] and PSPNet [23]. For the second stage, methods like skeleton or medial axis extraction with pre- and post-processing are often used to obtain the final road networks. However, recovering the correct connections and junctions of roads still remain challenging. This problem is critical since the road network is often used in routing and false breaks in the extraction lead to unacceptable results. This two-stage approach can potentially be fully automatized if training samples can be produced algorithmically.

To achieve the full automatization, one needs to have a direct reconstruction from images that may not be completely faithful but reliable enough to serve as the generator of good training samples. Then, one can iteratively use the technique to improve upon the training samples. This is what we achieve in this work for automatic road reconstruction. It turns out that our direct reconstruction method even improves over the state-of-the-art techniques for semi-automatic reconstructions by providing a more robust algorithm for the second stage. Our direct reconstruction method is a topology-based graph reconstruction algorithm. It uses the recent techniques of topological persistence [9] and discrete Morse theory [10] in topological data analysis. This topology-based approach for recovering hidden structures has been proposed and studied recently $[5,12,14,21]$. It has been applied to extracting graph-like structures from simulated dark matter density fields [17] and reconstructing road networks from GPS traces $[6,20]$. This 
discrete-Morse based graph reconstruction framework is clean both conceptually and implementation-wise. Most importantly, as it uses a global topological structure to make decisions (instead of using purely local information to decide whether a point is on or off the road), the algorithm is robust to noise, non-uniform sampling of the data, and reliable at recovering junctions. Very recently, this graph reconstruction algorithm has been further simplified, and theoretical guarantees of this graph reconstruction algorithm for the case when the signal prevails noise have been provided [7].

Contributions: Our contribution is twofold. (1) First, in a semiautomatic framework, we apply the discrete-Morse based graph reconstruction algorithm on the segmented satellite images obtained by a CNN. This, of course, requires user provided training samples to train the $\mathrm{CNN}$. We show that this leads to reconstructions with better network connectivity and less noise compared to some existing state-of-the-art technique. (2) More importantly, next, in a fully automatic framework, we develop a novel method to leverage the power of the discrete-Morse based graph reconstruction algorithm to train a CNN from a collection of images without labelled data so that it can produce segmentation for new images. To elaborate, we start with running the graph reconstruction algorithm on the raw satellite images to obtain some initial reconstructions. We then put the pixels from reliable branches of the output graph as positive and others as negative to create the labels for the training, and produce an intermediate $\mathrm{CNN}$ classifier. We predict the segmented images for the training set using this intermediate $\mathrm{CNN}$ and then repeat the same process on the output to gradually improve the $\mathrm{CNN}$. Our experiment shows that after several iterations of training, the labels computed from the graph reconstruction algorithm become less noisy and the performance of the classifier improves significantly. If we relax the condition slightly and assume that we know the labels for only $10 \%$ of the train set, we can incorporate this partially labelled data into our framework, and the performance of the classifier becomes even better. We experiment on datasets from SpaceNet Challenge [19] and compare our results with the results of the winner's algorithm, using the APLS score ([19]) as well as another metric which we call average Hausdorff distance. Our reconstructions tend to have better connectivity and are less noisy and we show promising experimental results of this new framework on datasets from SpaceNet Challenge.

Organization: Section 2 introduces our semi-automatic framework and fully automatic framework. Section 3 provides experiment results for both frameworks. A full version of this abstract is in [8].

\section{APPROACHES}

On the high level, the road network reconstruction from satellite images framework has two stages: We apply the simplified version of the discrete-Morse based graph reconstruction algorithm MorseGraphRecon() [7] to extract road networks from the segmented images (sometimes called "road masking" in the literature). Our approach mostly uses this reconstruction algorithm as a black box, interested readers should see [7] for more details.

\subsection{Semi-automatic framework}

The semi-automatic framework follows the high-level two-stage approach: In the first stage, we train a $\mathrm{CNN}$ using training images

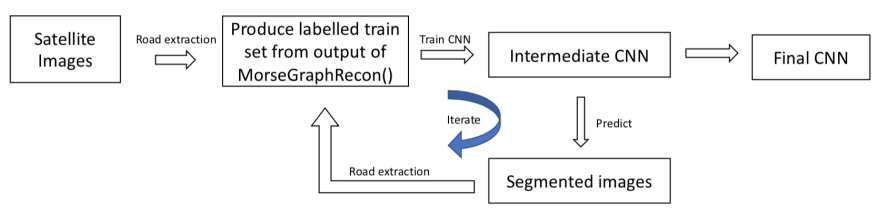

Figure 1: Pipeline for Stage 1 (CNN training) for our fully automatic framework. Note that no input satellite image has labels for roads!

consisting of ground-truth roads labeled. We feed raw satellite images to this trained $\mathrm{CNN}$ to obtain segmented images. In the second stage, we apply the discrete-Morse based graph reconstruction algorithm to extract the road-network from the segmented image. For the second stage to work more accurately, we need to detect road ends called "tips" in the segmented images obtained in the first stage. We take advantage of the $\mathrm{CNN}$ to add a simple "tipdetection" stage that enhances the segmented images. For details of reconstructing tips, see the full version [8].

CNN architecture. We use the architecture from the winner's approach of the Spacenet Challenge [2]. It uses resnet34 [13] as encoder and unet-like [15] decoder.

\subsection{Fully Automatic Framework}

The ground truth labeling used in the semi-automatic framework is itself a graph like structure. So we propose to create the labels using the discrete-Morse based graph reconstruction algorithm without the knowledge of the ground truth. These labels are used to train a $\mathrm{CNN}$ for the image segmentation. The segmented images are again labeled by the output of the graph reconstruction algorithm and fed to the $\mathrm{CNN}$ for training purpose. A few iterations of training and labeling improves the quality of the image segmentation significantly as our experiments show. This framework is particularly useful when there is no or very few labelled data to begin with.

Our framework can deal with the following two scenarios. We perform label-free learning when we do not have ground truth roads for any input satellite images to begin with. We perform partially-labeled learning when we have a small fraction of images (say $10 \%$ of training set) with road labels.

Label-free case. We describe the framework for the label-free case, and the partially-labeled case can be handled by a slight modification of it. The high-level pipeline of Stage 1 (training a CNN for segmenting an input image) is in Figure 1. Given an input set of raw satellite images (with no labels), we split it into the training and testing sets, denoted by $I_{0}^{t r}$ and $I_{0}^{\text {test }}$, respectively. We run algorithm MorseGraphRecon() on each image from $I_{0}^{t r}$, and let $\widehat{G}$ be its corresponding output. We use a large threshold for simplification in algorithm MorseGraphRecon() so as to generate a reconstruction of the more reliable part of the input. Then we label pixels on $\widehat{G}$ as positive and pixels on the complement of $\widehat{G}$ as negative. Next we train the CNN classifier with those labeled pixels, and this is our first classifier $C_{0}=$ MorseLabelTrain $\left(I_{0}^{\text {tr }}\right)$ (shown in Algorithm 1).

Now feeding each original training image from $I_{0}^{t r}$ to $C_{0}$ returns a collection of segmented images $I_{1}^{t r}$, where in each image, every 


\begin{tabular}{|l|l|}
\hline Algorithm 1: MorseLabelTrain $(I)$ \\
\hline \multicolumn{2}{|c|}{ Data: Images $I$} \\
Result: Classifier $C$ \\
1 \\
begin \\
2 & Compute the triangulation $K$ of $I$ and take pixel values as \\
& road-likehood map $\rho$ \\
3 & $\widehat{G}=$ MorseGraphRecon $(K, \rho, \delta)$ \\
4 & Label pixels on $\widehat{G}$ as positive, pixels on the complement of \\
& $\widehat{G}$ as negative \\
5 & Train a CNN classifier $C$ by above features \\
6 & return $C$ \\
\hline
\end{tabular}

pixel has a value reflecting the likelihood of it being positive (on the road). We repeat the steps with images in $I_{1}^{t r}$ and obtain a new CNN classifier $C_{1}=$ MorseLabelTrain $\left(I_{1}^{t r}\right)$. In a generic $i$-th iteration of this process, feeding the training images $I_{i}^{t r}$ to $C_{i}$ returns segmented images $I_{i+1}^{t r}$, which we use to train a new CNN classifier $C_{i+1}=$ MorseLabelTrain $\left(I_{i+1}^{t r}\right)$. The process terminates when the segmented images $I_{i}^{t r}$ undergo little changes over iterations.

Partially-labelled case. For this scenario, we start training the $\mathrm{CNN}$ classifier using only the labelled training images to obtain $C_{0}$. In each of the subsequent iteration $i>0$, we use both the labels computed from the segmented images $I_{i}^{t r}$ at this iteration, as well as the original labels from the ground truth.

\section{EXPERIMENTS}

Datasets. We consider data from the SpaceNet Challenge 3 [19]. It includes four cities: Las Vegas, Paris, Shanghai and Khartoum. We only use the RGB 16-bit geotiff in our experiments. Each image from the dataset covers $400 \mathrm{~m}$ by $400 \mathrm{~m}$ with a size of $1300 \mathrm{px}$ by $1300 \mathrm{px}$. The ground truth for each image is a graph representing the centerline of the roads. The width of the roads in the masks is 4 meters. To evaluate the results, we need to compare the proposed graphs with the ground truth. So we only take the train set from this challenge (since ground truth is only known for this set).

Metrics. We use the Average Path Length Similarity (APLS) [19] and average Hausdorff distance to evaluate the results. APLS is the metric used for evaluation in SpaceNet Challenge 3, it may not be accurate when the size of the graph is small. To obtain a more comprehensive picture, we also use another metric.

Definition 3.1. Let $G_{1}=\left(V_{1}, E_{1}\right)$ and $G_{2}=\left(V_{2}, E_{2}\right)$ be two input graphs. For $a, b \in V_{1}$ where path $(a, b)$ exists in $G_{1}$, let $a^{\prime}$ (resp. $b^{\prime}$ ) denote the closet node to a (resp. to $b$ ) in $G_{2} . L(\cdot, \cdot)$ denote the length of the shortest path. First we define the cost of path $(a, b)$ :

$$
c(a, b)= \begin{cases}\min \left\{1, \frac{\left.\left|L(a, b)-L\left(a^{\prime}, b^{\prime}\right)\right|\right)}{L(a, b)}\right\}, & \text { if path }\left(a^{\prime}, b^{\prime}\right) \text { exists } \\ 1, & \text { otherwise }\end{cases}
$$

We next define $C\left(G_{1}, G_{2}\right)=1-\frac{1}{N} \sum c(a, b)$ Where $N=$ \# unique paths in $G_{1}$, and we take the sum over all unique paths. Finally, the APLS score of $G_{1}$ and $G_{2}$ is defined to be the harmonic mean of $C\left(G_{1}, G_{2}\right)$ and $C\left(G_{2}, G_{1}\right): \operatorname{APLS}\left(G_{1}, G_{2}\right)=\frac{2}{\overline{C\left(G_{1}, G_{2}\right)}+\frac{1}{C\left(G_{2}, G_{1}\right)}}$.
Definition 3.2. Let $G_{1}$ and $G_{2}$ be two graphs; $P_{1}$ the point set sampled from $G_{1} ; P_{2}$ the point set sampled from $G_{2}$; and d denotes the Euclidean distance. The one-directional Hausdorff distance is:

$$
S_{H A}\left(G_{1}, G_{2}\right):= \begin{cases}\frac{1}{\left|P_{1}\right|} \sum_{p \in P_{1}} d\left(p, P_{2}\right), & G_{1} \neq \emptyset \text { and } G_{2} \neq \emptyset \\ M A X, & \text { Only one graph is empty }\end{cases}
$$

$S_{H A}\left(G_{1}, G_{2}\right)$ is set to 0 if both $G_{1}$ and $G_{2}$ are empty. MAX is a specific maximum value. We set $S_{H}\left(G_{1}, G_{2}\right)=S_{H A}\left(G_{1}, G_{2}\right)+S_{H A}\left(G_{2}, G_{1}\right)$ as the final average Hausdorff distance between $G_{1}$ and $G_{2}$.

Parameters. In the entire pipeline, the persistence threshold $\delta$ (for the discrete-Morse based reconstruction algorithm) and the arc-intensity threshold $\tau$ (used to further remove noisy arcs during the post-processing) affect the results most. See the full version [8] for the parameter tuning.

\subsection{Semi-automatic reconstruction results}

\begin{tabular}{|c|c|c|c|c|}
\hline & \multicolumn{2}{|c|}{ APLS } & \multicolumn{2}{c|}{$S_{H}$} \\
\hline & Buslaev[1] & ours & Buslaev[1] & ours \\
\hline AOI_2 & 0.8211 & $\mathbf{0 . 8 2 7 8}$ & 18.3539 & $\mathbf{1 7 . 7 8 4 1}$ \\
\hline AOI_3 & 0.5848 & $\mathbf{0 . 6 3 2 4}$ & 291.0188 & $\mathbf{2 8 9 . 9 5 3 2}$ \\
\hline AOI_4 & 0.6630 & $\mathbf{0 . 6 6 3 2}$ & 69.5775 & $\mathbf{6 8 . 9 5 9 6}$ \\
\hline AOI_5 & 0.6069 & $\mathbf{0 . 6 4 7 7}$ & 44.4201 & $\mathbf{4 1 . 6 0 3 7}$ \\
\hline
\end{tabular}

Table 1: APLS score and average Hausdorff distance for the test set. The $M A X$ value used for average Hausdorff distance is 500 pixels, the size of each image is $1300 \mathrm{px}$ by $1300 \mathrm{px}$.

We compare our framework with the method of the winner of SpaceNet Challenge 3 [1]. Buslaev's algorithm tends to have more extra branches, and worse connectivity. Tables 1 shows the scores under the two metrics. Each score is an average of scores for all test images. Table 2 shows the running time. Our framework gives better score under both two metrics and all four datasets. See the full version [8] for more details.

\begin{tabular}{|c|c|c|c|}
\hline & Train & Test & Road extraction \\
\hline AOI_2 & $2 \times 242 m$ & $8 m \times 2$ & $22 m \times 9$ \\
\hline AOI_3 & $77 m \times 2$ & $2 m \times 2$ & $6 m \times 9$ \\
\hline AOI_4 & $293 m \times 2$ & $10 m \times 2$ & $27 m \times 9$ \\
\hline AOI_5 & $71 m \times 2$ & $2 m \times 2$ & $6 m \times 9$ \\
\hline
\end{tabular}

Table 2: The running time: $m$ stands for minutes; $2 \times$ means it will be run twice (for both road and tip detections). $9 \times$ comes from the tuning of the parameters.

\subsection{Fully automatic reconstruction results}

In the following experiments, we randomly select 200 images as the training set $I_{0}^{t r}$, and 50 images as the test set $I_{0}^{\text {test }}$ for each dataset. We evaluate the method by computing the APLS scores on the original test set after each iteration. We initialize our fully automatic approach by converting each RGB image to grayscale and then applying a Gaussian filter. One could potentially use other image processing methods to further pre-process it. When applying the graph reconstruction algorithm, we use the same parameters used in Section 3.1. 
Alternative method for centerline detection. To show that the discrete-Morse based graph reconstruction algorithm is important for our fully-automatic training framework, we develop the following alternative scheme SkeletonLabelTrain() as a baseline to compare: the graph reconstruction algorithm is replaced with the Buslaev's [1] skeleton extraction algorithm (as described in Section 3.1). Note that this skeleton extraction used in [1] is not designed to work directly on the raw satellite images. Hence to improve the performance of this the baseline method SkeletonLabelTrain(), we will still first use the discrete-Morse graph reconstruction algorithm (or if there are partially-labelled data, using those first) at the beginning of the training process, and switch to the skeleton extraction algorithm only after a few iterations.

Results for label-free case. We show results here for dataset AOI_2, which is a cleaner dataset from SpaceNet Challenge. Our new fullyautomatic framework is less effective on AOI_5, which is much more noisy; however, we will show later that, with $10 \%$ labelled images, it can obtain reasonable results on the challenging AOI_5 dataset as well. In Figure 2, we show the APLS-score for test images using the CNN $C_{i}$ learned at the $i$-th iterations, as $i$ increases (the average Hausdorff distance shows a similar trend). In particular, $I_{i}^{\text {test }}(i>0)$ represents the output reconstructed from the segmented images of the set $I_{0}^{\text {test }}$ using the trained CNN $C_{i-1}$. In Figure 3, we show an example of the reconstructed graph using the $\mathrm{CNN}$ from different iterations of our fully automatic training process: observe that at the beginning, only part of signals are captured. Subsequently, the classifier becomes better and more and more signals are captured.

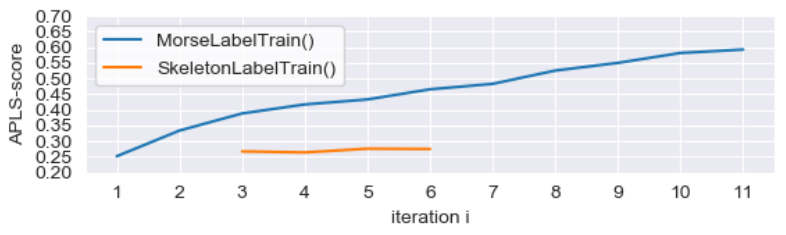

Figure 2: APLS-score for testing images, based on our labelfree framework (MorseLabelTrain()), compared to the alternative method SkeletonLabelTrain(). The first two iterations for SkeletonLabelTrain() is done by MorseLabelTrain() and thus are not shown. After 6 iterations, the score does not improve for SkeletonLabelTrain() any more.

With 10\% ground truth. Now we use a small set of labelled data: Specifically, we assume that only $10 \%$ images (i.e, 20 images) have labels (i.e, ground-truth roads given). For MorseLabelTrain(), all scores improve. It is important to note that with only $10 \%$ labeledimages, we can now also handle the challenging AOI_5 dataset, and achieve an APLS-score of 0.607. (For the case of AOI_2, compared to the label-free case, the score of our new MorseLabelTrain() improves to 0.721 from 0.592 ). This iterative procedure does not seem to help SkeletonLabelTrain() much, with scores even getting worse for the noisy dataset AOI_5. See the full version [8] for details.

\section{REFERENCES}

[1] A. Buslaev. 2018. Spacenet round 3 winner. https://github.com/ SpaceNetChallenge/RoadDetector/tree/master/albu-solution. (2018).

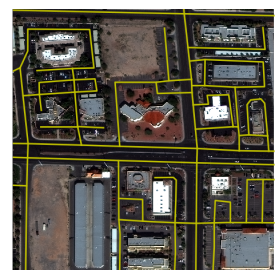

AOI_2 - Id: 323

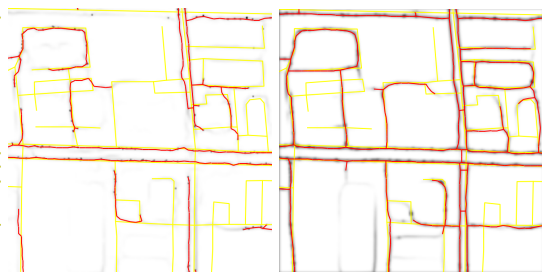

$I_{1}^{t e \cdot 0.0653 / 57.0400} I_{11}^{t e} \mathbf{0 . 5 5 6 4} / 30.3855$
Figure 3: Left: raw satellite image (ground truth in yellow, even though it is not used!). Middle / right: the reconstructed graph using $\mathrm{CNN}$ after the first and the 11-th iterations.

[2] A. Buslaev, S. Seferbekov, V. Iglovikov, and A. Shvets. 2018. Fully convolutional network for automatic road extraction from satellite imagery. In The IEEE Conference on Computer Vision and Pattern Recognition (CVPR) Workshops.

[3] D. Ciresan, A. Giusti, L. M. Gambardella, and J. Schmidhuber. 2012. Deep neural networks segment neuronal membranes in electron microscopy images. In Advances in neural information processing systems. 2843-2851.

[4] S. Das, TT Mirnalinee, and K. Varghese. 2011. Use of salient features for the design of a multistage framework to extract roads from high-resolution multispectral satellite images. IEEE transactions on Geoscience and Remote sensing 49, 10 (2011), 3906-3931.

[5] O. Delgado-Friedrichs, V. Robins, and A. Sheppard. 2015. Skeletonization and Partitioning of Digital Images Using Discrete Morse Theory. IEEE Trans. Pattern Anal. Machine Intelligence 37, 3 (March 2015), 654-666.

[6] T. K. Dey, J. Wang, and Y. Wang. 2017. Improved road network reconstruction using discrete morse theory. In Proc. 25th ACM SIGSPATIAL. ACM, 58.

[7] T. K. Dey, J. Wang, and Y. Wang. 2018. Graph Reconstruction by Discrete Morse Theory. In Proc. 34th Sympos. Comput. Geom. 31:1-31:15.

[8] T. K. Dey, J. Wang, and Y. Wang. 2019. Road Network Reconstruction from satellite images with Machine Learning Supported by Topological Methods. arXiv preprint arXiv:1909.06728 (2019).

[9] H. Edelsbrunner and J. Harer. 2010. Computational Topology : an Introduction. American Mathematical Society. I-XII, 1-241 pages.

[10] R. Forman. 1998. Morse theory for cell complexes. Advances in mathematics 134, 1 (1998), 90-145.

[11] X. Gu, A. Zang, X. Huang, A. Tokuta, and X. Chen. 2015. Fusion of color images and LiDAR data for lane classification. In Proc. 23rd ACM SIGSPATIAL. ACM, 69.

[12] A. Gyulassy, M. Duchaineau, V. Natarajan, V. Pascucci, E. Bringa, A. Higginbotham, and B. Hamann. 2007. Topologically Clean Distance Fields. IEEE Trans. Visualization Computer Graphics 13, 6 (Nov 2007), 1432-1439.

[13] K. He, X. Zhang, S. Ren, and J. Sun. 2016. Deep residual learning for image recognition. In Proc. of the IEEE conference on computer vision and pattern recognition. 770-778.

[14] V. Robins, P. J. Wood, and A. P. Sheppard. 2011. Theory and Algorithms for Constructing Discrete Morse Complexes from Grayscale Digital Images. IEEE Trans. Pattern Anal. Machine Intelligence 33, 8 (Aug 2011), 1646-1658.

[15] O. Ronneberger, P. Fischer, and T. Brox. 2015. U-net: Convolutional networks for biomedical image segmentation. In International Conference on Medical image computing and computer-assisted intervention. Springer, 234-241.

[16] W. Shi, Z. Miao, and J. Debayle. 2014. An integrated method for urban main-road centerline extraction from optical remotely sensed imagery. IEEE Transactions on Geoscience and Remote Sensing 52, 6 (2014), 3359-3372.

[17] T. Sousbie. 2011. The persistent cosmic web and its filamentary structure - I. Theory and implementation. 414 (June 2011), 350-383. arXiv:astro-ph.CO/1009.4015

[18] T. Sun, Z. Di, and Y. Wang. 2018. Combining Satellite Imagery and GPS Data for Road Extraction. In Proc. of the 2nd ACM SIGSPATIAL International Workshop on AI for Geographic Knowledge Discovery. ACM, 29-32.

[19] A. Van Etten, D. Lindenbaum, and T. M. Bacastow. 2018. SpaceNet: A Remote Sensing Dataset and Challenge Series. arXiv preprint arXiv:1807.01232 (2018).

[20] S. Wang, Y. Wang, and Y. Li. 2015. Efficient map reconstruction and augmentation via topological methods. In Proc. 23rd ACM SIGSPATIAL. ACM, 25.

[21] K. Weiss, F. Iuricich, R. Fellegara, and L. De Floriani. 2013. A primal/dual representation for discrete Morse complexes on tetrahedral meshes. In Computer Graphics Forum, Vol. 32. 361-370.

[22] A. Zang, R. Xu, Z. Li, and D. Doria. 2017. Lane boundary extraction from satellite imagery. In Proc. of the 1st ACM SIGSPATIAL Workshop on High-Precision Maps and Intelligent Applications for Autonomous Vehicles. ACM, 1.

[23] H. Zhao, J. Shi, X. Qi, X. Wang, and J. Jia. 2017. Pyramid scene parsing network. In IEEE Conf. on Computer Vision and Pattern Recognition (CVPR). 2881-2890. 